\title{
Impact of health education on knowledge and behaviours toward obstetric fistula among women of reproductive age in Uganda
}

Betty K. Nannyonga and Martin Singull

The self-archived postprint version of this journal article is available at Linköping University Institutional Repository (DiVA):

http://urn.kb.se/resolve?urn=urn:nbn:se:liu:diva-163442

N.B.: When citing this work, cite the original publication.

Nannyonga, B. K., Singull, M., (2020), Impact of health education on knowledge and behaviours toward obstetric fistula among women of reproductive age in Uganda, Applied Mathematics and Computation, 372, 124997. https://doi.org/10.1016/j.amc.2019.124997

Original publication available at:

https://doi.org/10.1016/j.amc.2019.124997

Copyright: Elsevier

http://www.elsevier.com/

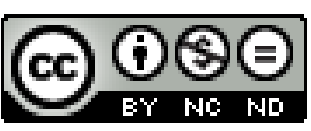




\title{
Impact of Health Education on Knowledge and Behaviors toward Obstetric Fistula among Women of Reproductive Age in Uganda
}

\author{
Betty K. Nannyonga ${ }^{\mathrm{a}, *}$, Martin Singull ${ }^{\mathrm{b}, *}$ \\ ${ }^{a}$ Department of Mathematics, School of Physical Sciences, College of Natural Sciences, Makerere University, \\ P.O. Box 7062, Kampala Uganda. \\ ${ }^{b}$ Department of Mathematics, Mathematical Statistics, Linköping University Sweden.
}

\begin{abstract}
Obstetric fistula is among the most severe maternal morbidities. It is caused directly by obstructed labour, and indirectly by social, political, and economic factors. Effects of obstetric fistula include chronic urinary incontinence. Urinary incontinence often leads to isolation and death if left untreated. Death of women during childbirth is detrimental to both social economic transformation and shift of poor economies to middle income status. In this study, we estimate the burden of obstetric fistula on Ugandan women, and the effect of educating these women on its causes. We hypothesise that symptomatic women seek treatment after interactions with a treated woman. We consider one cycle of fistula and seek to determine how many women will eventually seek treatment, when one woman is treated in a population. We further assume that each treated woman becomes the educational resource for more symptomatic women. We then invest in getting women into treatment, providing treatment, or educating them about prevention and treatment of obstetric fistula. Results show that for each investment strategy, there exists a unique maximal solution that remains bounded. It is also shown from the model that high investment rates leads to high treatments rates. However, the highest treatment output is obtained when the level of investment is $44.9 \%$. We also see from the results that for every one woman treated, 17 more seek treatment. However, as investment increases, the number of women that are eventually treated reduces due to lack of further investment into treatment and therefore no more women to treat. Results also show that educating women reduced fistula prevalence although the magnitude of reduction depended on the level of education coverage. These results show that an optimal strategy to reduce fistula prevalence would require achieving a balance between the two desirable but incompatible health outcomes. This will help maximise the total return or effectiveness under given circumstances such as continued women that develop fistula due to lack of skilled healthcare or lifetime incontinence due to lack of treatment. From this study we conclude that improving access to quality health education will contribute to the elimination of obstetric fistula in Uganda.
\end{abstract}

Keywords: Obstructed labour, Obstetric fistula, Vesicovaginal fistula, Rectovaginal fistula, Skilled health care, Reproductive health, Sexual health, Women's health 2008 MSC: 93A30, 92D25

\footnotetext{
*Betty Nannyonga

Email addresses: bnkivumbi@gmail.com (Betty K. Nannyonga), martin.singull@liu.se (Martin Singull)

URL: www.math.mak.ac.ug (Betty K. Nannyonga), www.liu.se (Martin Singull)
} 


\section{Introduction}

Health care services during pregnancy and childbirth are important for the survival and wellbeing of both mother and infant. Maternal health is a core dimension of the global health development agenda. In Uganda, maternal disorders are among the most important cause of death among women of reproductive age ( $15-49$ years old) [1]. Disease burden attributable to maternal complications is important despite significant declines in maternal mortality. The Government of Uganda prioritised maternal health in the national health agenda through the Roadmap for Accelerating the Reduction of Maternal and Neonatal Mortality and Morbidity in Uganda (2007 - 2015) and as a strategic and priority health care intervention area under the current Health Sector Development Plan (HSDP 2015/16 - 2019/2020). Although reduction in maternal mortality has been observed in sub-Saharan countries since the mid 2000s [2, 3], it is estimated that for every woman who died from maternal complications, another 20 women withstood serious maternal morbidity [4]. Of all maternal morbidities, obstetric fistula is one of the most debilitating conditions with the immediate consequence of chronic urinary and/or incontinence. Physical comorbidities, psychological distress, and social stigmatisation usually follow $[5,6,7,8,9]$.

Obstetric fistula is inextricably linked to biological, socioeconomic, and cultural factors that favour obstructed labor and caused by lack of, delay of, or inadequate emergency obstetric care $[7,10,11,12]$. Obstructed larour results in an abnormal opening between the vagina and the bladder (vesicovaginal fistula) or rectum (rectovaginal fistula), leading to continuous urinary or incontinence. Risk factors for obstetric fistula include duration of labor, provision of basic and emergency care to women during childbirth, mode and place of delivery $[13,14,15$, $16,17,18,19,20,21]$. Many facility-based studies provide insights into various attributes of fistula afflicted women [12] while comparative studies have aided in confirming the risk factors $[22,23,24]$, or develop prevention and treatment strategies [25]. Numerous studies have been carried out and aimed at reducing maternal mortality due to delay in seeking, reaching, and receiving adequate care $[21,26,27,28]$. Social, political, and economic causes that indirectly lead to the development of obstetric fistulae concern issues of poverty, malnutrition, lack of education, early marriage and childbirth (see Figure 1 ), the role and status of women in developing countries, harmful traditional practices, and lack of good quality or accessible maternal and health care $[9,18,23,29]$.

In addition to the despair from losing their child, the psychological consequences that women
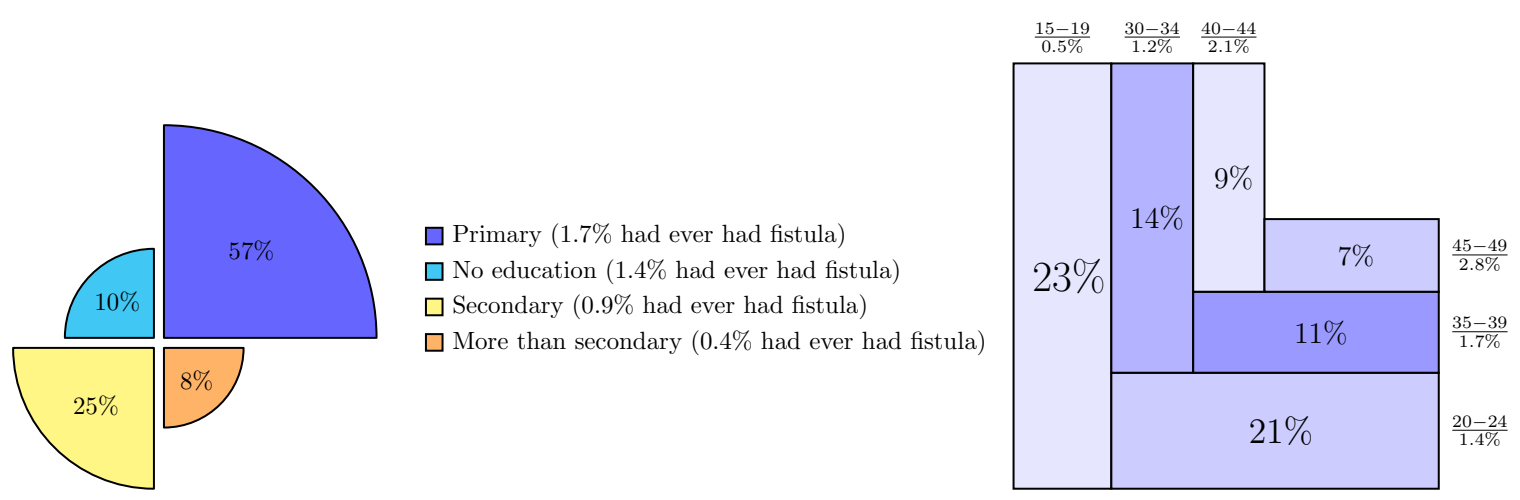

Figure 1: Figure showing the percentage distributions of women based on their level of education and age, together with what percentage of each group had ever had fistula symptoms 
with a fistula face are humiliation from their smell, and inability to perform their family roles $[30,31,32,33]$. They also endure repeated vaginal or urinary tract infections [31, 32, 34], irritation or pain in the vagina or surrounding areas [32,35,36], and pain during sexual activity $[32,35,36]$. As a result, they are often spurned from societies and have to deal with the physical and psychological problems of their condition [37, 38, 39, 40, 41, 42]. Due to ignorance and lack of sensitisation, they often do not know that help is available and are too ashamed to ask. This leads women to live with the condition for many years [43], often without any economic contribution to their families or community [38, 39, 41, 42, 44].

The aim of this study is to examine the association between education level and the prevalence of obstetric fistula in Ugandan women. The data we use is from the Uganda Bureau of Statistics, Uganda Demographic Health Survey carried out in fifteen regions of South Central, North Central, Kampala, Busoga, Bukedi, Bugisu, Teso, Karamoja, Lango, Acholi, West Nile, Bunyoro, Tooro, Kigezi and Ankole. It mainly considered at the age of the women at birth, whether they accessed antenatal care or not during pregnancy, reasons why they did not, whether they had birth at a health facility attended by a skilled provider, the women's level of education, wealth quintile, and their experiences with obstetric fistulas. To achieve our objective, the women are grouped into three categories: those with fistula symptoms, those that had it and got treated and the recovered. First we consider the case of one cycle without demographics to determine the burden in Uganda. We then repeatedly offer education to women in the reproductive age of $15-49$ to determine how it impacts the overall outcome in comparison to absence of education. Since education might take a longer period to realise benefits and its potential in the fight against obstructed labour, demographics are incorporated in the revised model but the population is kept constant. We look at three scenarios: no education to women, partial education or full education. The education offered is on the causes, dangers, and possible prevention of obstetric fistula. It also provides opportunities for symptomatic women such as treatment for the condition. To optimise health returns, an investment strategy is incorporated which provides the best way to prevent obstructed labour.

\section{Formulation of the model}

In this study one cycle of fistula is considered. Women who get fistula are treated and removed. We assume an ideal situation where treatment is available, and the reasons that led to the women to get fistula are not modelled. The overarching aim is to determine the burden of fistula when treatment for the symptomatic is available. To determine the fistula curve, we

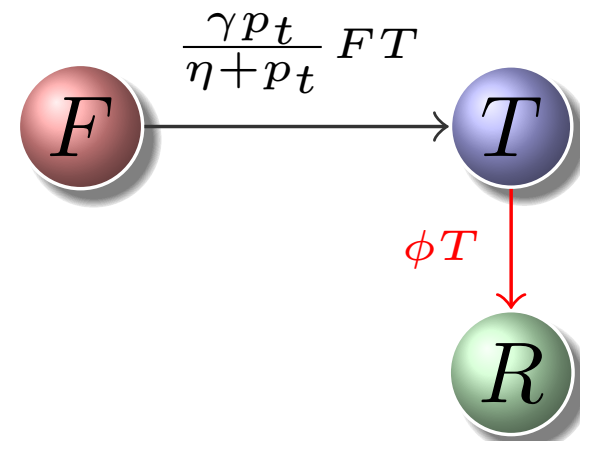

Figure 2: Flux diagram for one cycle of obstetric fistula 
make an assumption that there is a single reproductive cycle of obstetric fistula development, from pregnancy to obstructed labour, fistula development, and immediate operation. Since a single pregnancy cycle lasts nine months, vital dynamics are ignored. Further, an ideal situation was assumed and therefore no fistula induced deaths pre- or post treatment. This keeps the population of the symptomatic women constant within any year of consideration. The model is therefore an FTR where a woman gets fistula, is treated and removed, similar to the standard $S I R$ type. Without demographics, women with fistula $F$ can only leave this group through treatment and move to class $T$, then transition to $R$ after removal through recovery. Let the treatment rate be $\gamma$ and recovery rate $\phi$. We assume that treatment is dependent on interaction between the treated and the fistula symptomatic. We make this assumption such that transition from $F$ to $T$ is associated with the numerical response, which is the reproduction rate of fistula women as a function of how many are treated. It also depends on the half saturation constant $\eta$ which is the point where there is a $50 \%$ chance of a particular fistula woman to get treatment in a given cycle at a level of investment in treatment $p_{t}$. The dynamics described here are depicted in Figure 2.

From Figure 2, the corresponding model is given by

$$
\frac{d F}{d t}=-\frac{\gamma p_{t}}{\eta+p_{t}} F T, \quad \frac{d T}{d t}=\frac{\gamma p_{t}}{\eta+p_{t}} F T-\phi T, \quad \frac{d R}{d t}=\phi T,
$$

with initial conditions $F(0) \geq 0, T(0) \geq 0, R(0)=0$. It is noted that the total number of women affected by obstetric fistula in a single reproductive cycle is given by $N_{f}=F+T+R$. All parameters and state variables of the autonomous model (1) are non-negative for all time $t$ so the model can be studied in the invariant region: $\mathcal{G}=\left\{(\mathcal{F}, \mathcal{T}, \mathcal{R}) \in \mathbb{R}^{3}: F, T, R \geq 0\right\}$, where it is mathematically well-posed.

\subsection{Analysis of the model}

Given parameters $\frac{\gamma p_{t}}{\eta+p_{t}}$ and $\phi$, the model is solved for $F(t), T(t)$ and $R(t)$ which satisfy the system of ODEs in equation (1) with initial conditions $F(0), T(0)$ and and $R(0)$. The total population size $N_{f}=F(t)+T(t)+R(t)$ is constant since $R$ are the removed individuals either due to death, or recovery and they cannot get obstetric fistula again. Assume that $F(t)$ is monotonically decreasing towards $F_{\infty}=\lim _{t \rightarrow \infty} F(t)>0$ (Figure 3a). If $F(0) \leq \frac{\gamma p_{t}}{\eta+p_{t}}, T(t)$ monotonically decreases towards $T_{\infty}(t)=0$, and there is no fistula development. However, if $F(0) \geq \frac{\gamma p_{t}}{\eta+p_{t}}, \quad T(t)$ increases initially and later decreases to zero, and we have fistula in the population (Figure $3 b$ ).

For each initial condition $F(0) \geq 0, T(0) \geq 0$, and $R(0) \geq 0$, there exists a unique solution of the model in equation (1) defined on $t \in[0, \infty)$. Similarly, for each investment strategy $p_{t}$, there exists a unique solution on $t \in[0, \infty)$ (see Figure 3 ). Each component $F(t), T(t), R(t)$ of the solution is non-negative and $N_{f}=F(t)+T(t)+R(t)$ is constant. If $F(0)>0$ and $T(0)>0$, then $F(t)>0$ and $T(t)>0$ for all $t \in[0, \infty)$. The model is therefore well posed.

Proposition 2.1. There exists a unique maximal solution $\{F(t), T(t), R(t)\}$ that remains bounded on $t \in[0, b)$.

Proof: The vector field $f:[-\infty, \infty) \times \mathbb{R}^{3} \rightarrow \mathbb{R}^{3}$ is defined by $f(t, F, T, R)=\left(\begin{array}{c}-\frac{\gamma p_{t}}{\eta+p_{t}} F T \\ \frac{\gamma p_{t}}{\eta+p_{t}} F T-\phi T \\ \phi T\end{array}\right)$. 

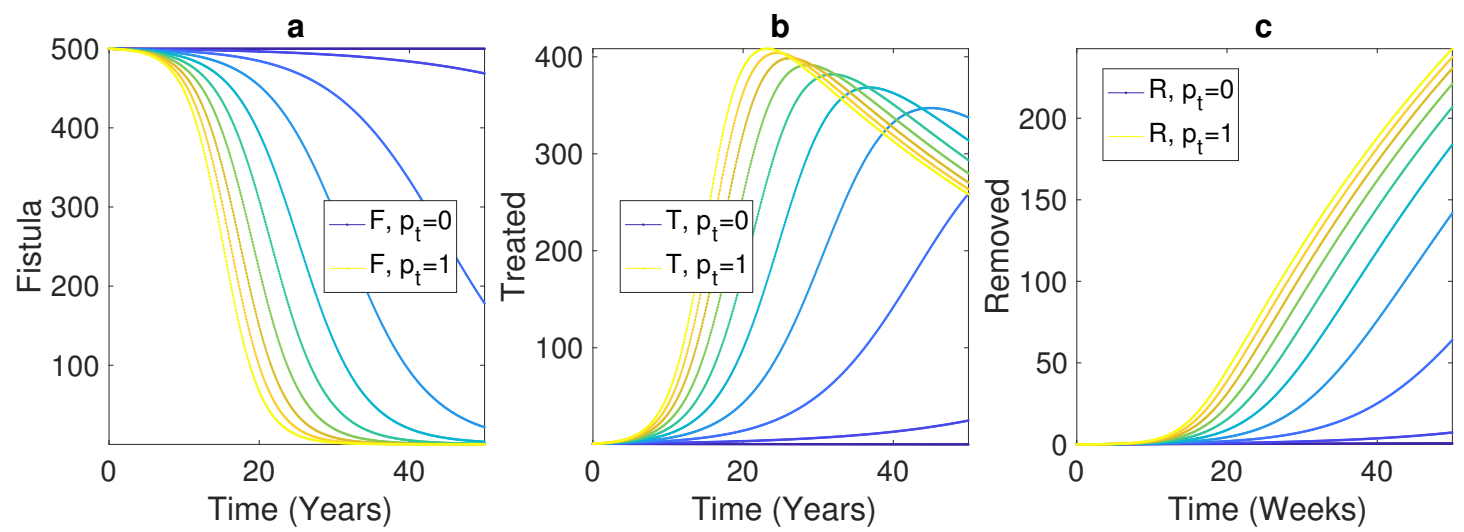

Figure 3: Evolution of the model with $\%$ investment in skilled care $p_{t} \in[0,1]$. Parameter values used are $\gamma=0.0017, \eta=\in[0,1]$ and $\phi=0.0192$. These parameters are estimated using data from Uganda

Since $f$ is continuous, there exists a maximal solution $\{F(t), T(t), R(t)\}$ defined on some open interval containing 0 by the maximum interval of existence theorem. In particular, the maximal solution is defined on $[0, b)$ for some $0<b \leq \infty$. Since the derivative of $f$ with respect to $(F, T, R)$ is $f(t, F, T, R)=\left(\begin{array}{ccc}-\frac{\gamma p_{t}}{\eta+p_{t}} T & -\frac{\gamma p_{t}}{\eta+p_{t}} F & 0 \\ \frac{\gamma p_{t}}{\eta+p_{t}} T & \frac{\gamma p_{t}}{\eta+p_{t}} F-\phi & 0 \\ 0 & \phi & 0\end{array}\right)$, then $f^{\prime}$ is continuous with respect to $(F, T, R)$ and so $f$ is locally Lipschitz continuous on $(-\infty, \infty) \times \mathbb{R}^{3}$. This means that the solution $(F(t), T(t), R(t))$ is uniquely defined on $[0, b)$.

It can be seen that $\frac{d F}{d t}+\frac{d F}{d t}+\frac{d R}{d t}=0$, and so $N_{f}=F(t)+T(t)+R(t)$ is a constant. From the first equation of system (1),

$$
F(t)=F(0) e^{-\frac{\gamma p_{t}}{\eta+p_{t}} \int_{0}^{t} T(t) d t} \geq 0, \text { for } t \in[0, b) .
$$

From the second equation of (1), we have

$$
T(t)=T(0) e^{\int_{0}^{t}\left(\frac{\gamma p_{t}}{\eta+p_{t}} F(t)-\phi\right) d t} \geq 0 .
$$

Therefore, $F(t)>0$ and $T(t)>0$ for $t \in[0, b)$ if $F(0)>0$ and $T(0)>0$. From the last equation of system (1), we have

$$
R(t)=R(0)+\phi \int_{0}^{t} T(t) d t \geq 0
$$

Hence the solution remains bounded on $t \in[0, b)$, i.e., $0 \leq F(t)+T(t)+R(t)=N_{f}$ for $t \in[0, b)$. Therefore, $b=\infty$.

Proposition 2.2. Whenever there is treatment, the final number of women with fistula is less than the initial number of symptomatic women; i.e., $F_{\infty}=\lim _{t \rightarrow \infty} F(t)<\frac{\gamma p_{t}}{\left(\eta+p_{t}\right) \phi}$.

Proof: We start this proof by noting that if $T(0)=0$, then $T(t)=0$ for all $t \geq 0$; i.e., if at time zero there is no treated woman in the population, then there will be no treatment for any symptomatic woman for all time $t>0$. If $F(0)=0$, then $F(t)=0$ for all 
$t \geq 0$ and so $\frac{d T}{d t}=-\phi T$ for all $t \geq 0$. Hence if $F(0)=0$ or $T(0)=0$, we have $T(t) \rightarrow 0$ as $t \rightarrow \infty$. This implies that whenever there is neither women with fistula nor women treated, it is impossible to have any women treated at any time $t>0$. Using a contradiction, let us assume that $F(0), T(0)>0$, and so $F(t), T(t)>0$ for all $t \geq 0$. Supposing that

$F_{\infty} \geq \frac{\gamma p_{t}}{\left(\eta+p_{t}\right) \phi}, \quad$ then $\frac{d T}{d t} \geq\left(\frac{\gamma p_{t}}{\eta+p_{t}}-\phi\right) T \geq 0$. Hence $T(t) \geq T(0)$ for all $t \geq 0$.

Recall that

$$
\frac{d F}{d t} \leq-\frac{\gamma p_{t}}{\eta+p_{t}} T(0) F, \quad \text { and so } F(t) \leq F(0) e^{-\frac{\gamma p_{t}}{\eta+p_{t}} T(0) t} \rightarrow 0 \quad \text { as } t \rightarrow \infty,
$$

contradicting the assumption that $F_{\infty} \geq \frac{\gamma p_{t}}{\left(\eta+p_{t}\right) \phi}$. We therefore must have $F_{\infty}<\frac{\gamma p_{t}}{\left(\eta+p_{t}\right) \phi}$.

Proposition 2.3. There are always some women with obstetric fistula left un treated at the end of the cycle.

Proof: Let $(F(t), T(t))$ be a solution of equation (1) with $F(0)>0$ and $T(0)>0$. Then limit $F_{\infty}=\lim _{t \rightarrow \infty} F(t)$ exists and

$$
0<F_{\infty}<\frac{\gamma p_{t}}{\left(\eta+p_{t}\right) \phi}
$$

This means that there are always some women with obstetric fistula left untreated and are less than $\frac{\gamma p_{t}}{\left(\eta+p_{t}\right) \phi}$.

\subsection{Basic reproductive ratio $\mathcal{R}_{0}$}

The basic reproductive ratio $\mathcal{R}_{0}$ for a fistula cycle in presence of treatment can be thought of as the number of fistula recovered women generated from one treated woman over the course of their recovery period, in an otherwise treatment-free population. The basic reproductive ratio is affected by several factors including the duration for lack of treatment of symptomatic women, treatment rates, and the number of women with fistula symptoms in the population that the treated women are in contact with. For continued treatment the number of treated women must increase - i.e., $\frac{d T}{d t}>0$ at all times. Using the second equation of model (1) it is observed that $\frac{d T}{d t}>0$ whenever $\frac{\gamma p_{t}}{\left(\eta+p_{t}\right) \phi} F_{0}>1$. Let

$$
\mathcal{R}_{0}=\frac{\gamma p_{t}}{\left(\eta+p_{t}\right) \phi} F_{0}
$$

where $F_{0}=N_{f}$.

Proposition 2.4. If a treated woman fails to convince a single symptomatic woman to seek treatment then fistula remains in the population.

Proof: If $\mathcal{R}_{0} \leq 1$, then $T(t)$ is strictly decreasing towards 0 as $t \rightarrow \infty$ and $T_{\max }=T(0)$, such that $F_{\infty} \rightarrow F(0)$ as $T(0) \rightarrow 0$. This is so since if $0<F(0) \leq \frac{\gamma p_{t}}{\left(\eta+p_{t}\right) \phi}$ then $F(t)<\frac{\gamma p_{t}}{\left(\eta+p_{t}\right) \phi}$ for $t>0$ since $F(t)$ is strictly decreasing. Hence $\frac{d T}{d t}=\left(\frac{\gamma p_{t}}{\eta+p_{t}}-\phi\right)<0$ for $t>0$, and so $T(t)$ is strictly decreasing. Therefore, $T_{\max }=T(0)$. The limit $\lim _{t \rightarrow \infty} T(t)=0$, such that $F_{\infty} \rightarrow F(0)$ as $T(0) \rightarrow 0$. This means that there are no women treated. 
Proposition 2.5. If a treated woman convinces a single symptomatic woman to seek treatment then the prevalence of fistula will be reduced.

Proof: If $\mathcal{R}_{0}>1$, then $T(t)$ is initially increases and then decreases towards 0 as $t \rightarrow \infty$ and $T_{\max }$ stays bounded away from 0 as as $T(0) \rightarrow 0$, and $F_{\infty}<\frac{\gamma p_{t}}{\left(\eta+p_{t}\right) \phi}<F(0)$ as $T(0) \rightarrow 0$.

The relationship between the basic reproductive ratio $\mathcal{R}_{0}$, the percent investment into treatment of symptomatic women $p_{t}$, the eventual number of women with fistula $F(t)$, treated $T(t)$, and the recovered $R(t)$ at any time $t$ is obtained and shown in Figure 4. It is observed from the figure that low investment rates lead to low treatment rates and high investment rates give rise to low fistula rates. The highest treatment output is obtained when the level of
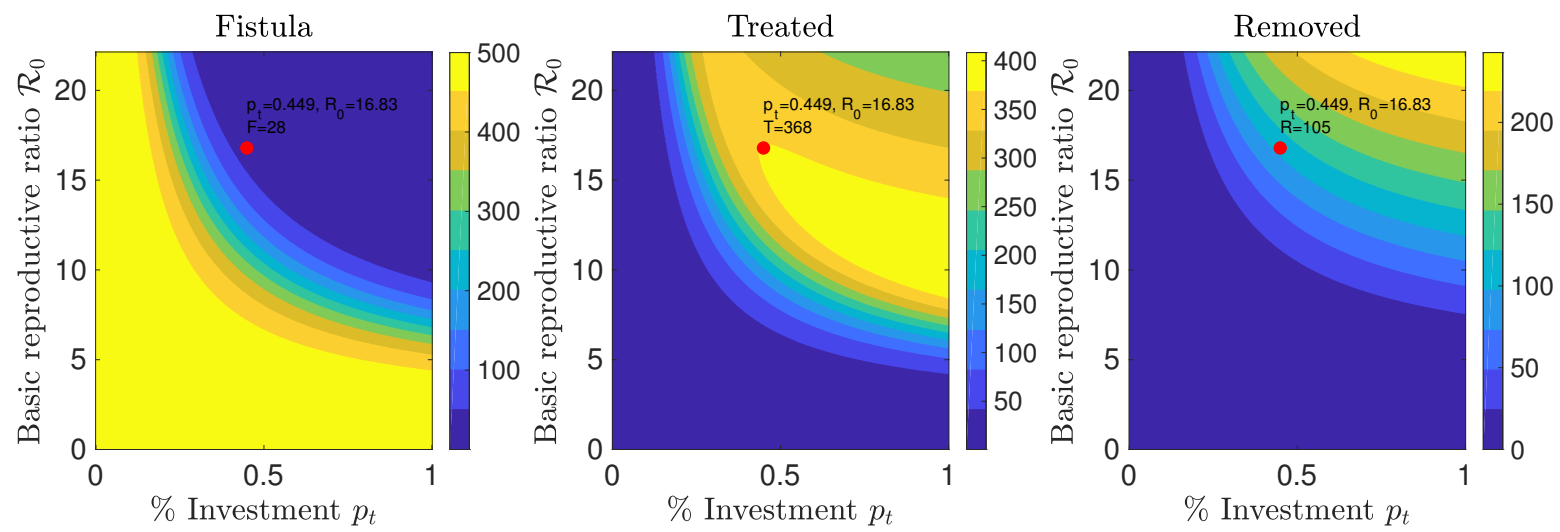

Figure 4: Plot of Fistula women $F$, the treated $T$, removed $R$ with changes in $\mathcal{R}_{0}$ as $p_{t}$ increases

investment is $p_{t}=0.449$. At this point, the basic reproductive ratio $\mathcal{R}_{0} \approx 17$. This threshold value implies that each treated woman leads to 17 new fistula women to seek treatment. The corresponding values of $F, T$ and $R$ at this point are 28,368 and 105 respectively. The initial values used to simulate the model are respectively $500,1,0$ for $F, T, R$. At the optimal treatment point, we have respective values 28,368 and 105 totalling to 501 . This implies that women are not dying from fistula but just transitioning through the different states of the model and the population remains constant at all times. This result is in agreement with Propositions 2.1, 2.2 and 2.3.

\subsection{Size of fistula burden}

We can obtain the epidemic curve, which is the rate at which women recover from obstetric fistula upon operation. In model (1), this is the equation $R$ for the removed cases. Recall that $N_{f}=F+T+R$. We can thus write $\frac{d R}{d t}=\phi T$, as

$$
\frac{d R}{d t}=\phi\left(N_{f}-F-R\right)
$$

Dividing the first equation of $(1)$ by the third gives $\frac{d F}{d R}=-\frac{\gamma p_{t}}{\left(\eta+p_{t}\right) \phi} F$, where $\log \left(\frac{F_{0}}{F}\right)=$ $\frac{\gamma p_{t}}{\left(\eta+p_{t}\right) \phi} R$, since $R_{0}=0$. Therefore $\frac{d R}{d t}=\phi\left[N_{f}-F_{0} e^{-\frac{\gamma p_{t}}{\left(\eta+p_{t}\right) \phi} R}-R\right]$. It is impossible to obtain $R$ as an explicit function of $t$. We therefore expand the exponential term in powers of $\frac{\gamma p_{t}}{\left(\eta+p_{t}\right) \phi} R$, assuming that the expression is very small compared to unity. 
Therefore,

$$
\frac{d R}{d t}=\phi\left\{N_{f}-F_{0}+\left[\frac{\gamma p_{t}}{\left(\eta+p_{t}\right) \phi} F_{0}-1\right] R-\frac{F_{0}}{2}\left[\frac{\gamma p_{t}}{\left(\eta+p_{t}\right) \phi} R\right]^{2}\right\} .
$$

But $N_{f}-F_{0}=T_{0}$, where $T_{0}$ is very small. Thus, although we assumed that $\frac{\gamma p_{t}}{\left(\eta+p_{t}\right) \phi} R \lll 1$, its square may not be as small compared to $\left[\frac{\gamma p_{t}}{\left(\eta+p_{t}\right) \phi} F_{0}-1\right] R$. The solution of equation (4) is given by

$$
\begin{aligned}
& R(t)=\frac{1}{F_{0}}\left[\frac{\left(\eta+p_{t}\right) \phi}{\gamma p_{t}}\right]^{2}\left\{\frac{\gamma p_{t}}{\left(\eta+p_{t}\right) \phi} F_{0}-1+\left\{\left(\frac{\gamma p_{t}}{\left(\eta+p_{t}\right) \phi} F_{0}-1\right)^{2}\right.\right. \\
& \left.+2 F_{0} T_{0}\left(\frac{\gamma p_{t}}{\left(\eta+p_{t}\right) \phi}\right)^{2}\right\}^{\frac{1}{2}} \tanh \left(\frac { 1 } { 2 } \left\{\left(\frac{\gamma p_{t}}{\left(\eta+p_{t}\right) \phi} F_{0}-1\right)^{2}\right.\right. \\
& \left.+2 F_{0} T_{0}\left(\frac{\gamma p_{t}}{\left(\eta+p_{t}\right) \phi}\right)^{2}\right\}^{\frac{1}{2}} \phi t \\
& \left.\left.-\tanh ^{-1}\left(\frac{\frac{\gamma p_{t}}{\left(\eta+p_{t}\right) \phi} F_{0}-1}{\left\{\left(\frac{\gamma p_{t}}{\left(\eta+p_{t}\right) \phi} F_{0}-1\right)^{2}+2 F_{0} T_{0}\left(\frac{\gamma p_{t}}{\left(\eta+p_{t}\right) \phi}\right)^{2}\right\}^{\frac{1}{2}}}\right)\right)\right\} .
\end{aligned}
$$

At the end of the cycle, the number of women recovered from obstetric fistula is given by

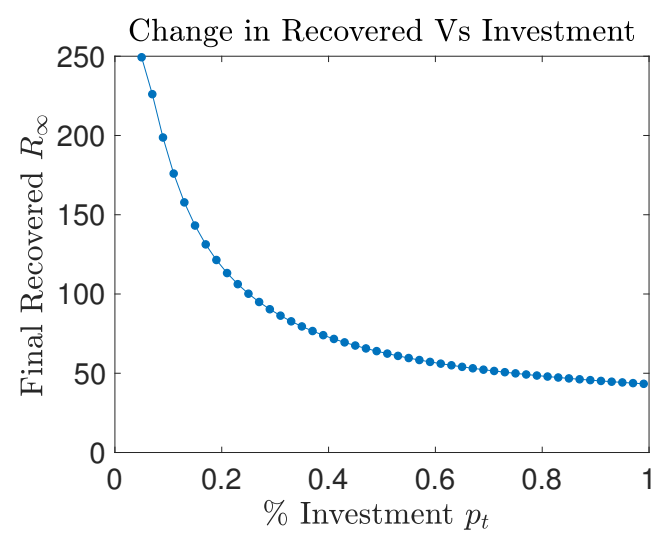

Figure 5: Plot of the final number of women recovered from fistula with corresponding investment into treatment $p_{t}$.

$$
R_{\infty}=\frac{2}{F_{0}} \frac{\left(\eta+p_{t}\right) \phi}{\gamma p_{t}}\left(F_{0}-\frac{\left(\eta+p_{t}\right) \phi}{\gamma p_{t}}\right)
$$

where we have neglected $T_{0}$ since it is small compared to $F_{0}$. We see from equation (6) that when $F_{0}=\frac{\left(\eta+p_{t}\right) \phi}{\gamma p_{t}}$, i.e., when $\mathcal{R}_{0}=1$, there is no recovery from fistula. Plotting the changes in the $\%$ investment $p_{t}$ against number of women recovered $R$ with increasing investment gives Figure 5. Note that as investment increases, the number of women that eventually recover reduces. This is because whenever all investment is in treatment, it implies that there 
is no effort getting women into treatment and therefore no more women to treat. An optimal strategy requires to achieve a balance between the two desirable but incompatible health outcomes. A tradeoff is needed where we reduce investment into antenatal in exchange to increase investment in treatment without foregoing any of the desirable outcomes. This will help maximise the total return or effectiveness under given circumstances such as continued women that develop fistula due to lack of skilled healthcare or lifetime incontinence due to lack of treatment.

\section{Education for fistula women}

In this section, we study the model while incorporating education for pregnant women. The pulse education strategy can be used to eradicate fistula by repeatedly educating women at risk, over the age range of 15 - 49, until cases of obstructed labor stop. Uganda has high the fertility rates (5.82) [46], and the mean age at first birth is 18.2 [47]. From the 2016 Uganda Demographic Health Survey, the total wanted fertility rate was 4.3 , which is lower than the actual fertility rate. Wanted fertility rate is the average number of children a woman would have by the end of her childbearing years if she bore children at the current agespecific fertility rates, excluding unwanted births. Therefore on average, women in Uganda are currently having one child more than they want. From the data, 97\% of the women received antenatal care (ANC) from a skilled provider during their most recent pregnancy. However, only $29 \%$ of them had their first ANC visit during the first trimester of pregnancy and only $60 \%$ completed at least four ANC visits. The median gestational age at which women made their first ANC visit was 4.7 months. This implies that women start attending ANC almost halfway through the overall gestation period; earlier attendance would provide a better opportunity to diagnose possible complications or pregnancy-related problems at the early stages of pregnancy. Further, the data shows that more than 7 in 10 live births were delivered in a health facility $(73 \%)$ and with skilled birth attendance $(74 \%)$ and the likelihood of seeing a doctor doubled among women with a secondary education (13\%) as compared with women with no education or a primary education (6-7\%) and doubled again among women with more than a secondary education $(28 \%)$.

The question we need to answer in this section is how can fistula control measures be efficiently applied so as to minimise prevalence, minimise the incidence of fistula or even eradicate obstructed labour all together. To do this, we revise equation (1) and incorporate education for women about effects of not attending antenatal care as soon as they realise that they are pregnant. It should be noted that the problems given by women of why they did not access heath care could be diverted if the women were empowered through education. Problems such as "not wanting to go alone" or "getting permission," could all be averted. The form of control applied here is simply moving a proportion $e$ of the women who would have gotten fistula to the removed class $R$ through empowerment/education. This would reduce the influx to $F$ and directly reduce in flow to $T$ and increase $R$. Reverting to $N_{f}=F+T+R$, with a specific size of $N_{f}$ at any given instant, increasing $R$ reduces both $F$ and $T$ and indirectly reduce the economic burden of fistula to Uganda.

To highlight the importance of education (number of years in school), we simulate the number of years spent in school with indicators such as child mortality, GDP and fertility rates (average child per woman). The data used was obtained from Gapminder.org and the results are shown in Figures 6 . Uganda is highlighted in bold red and it is observed from Figures 6 

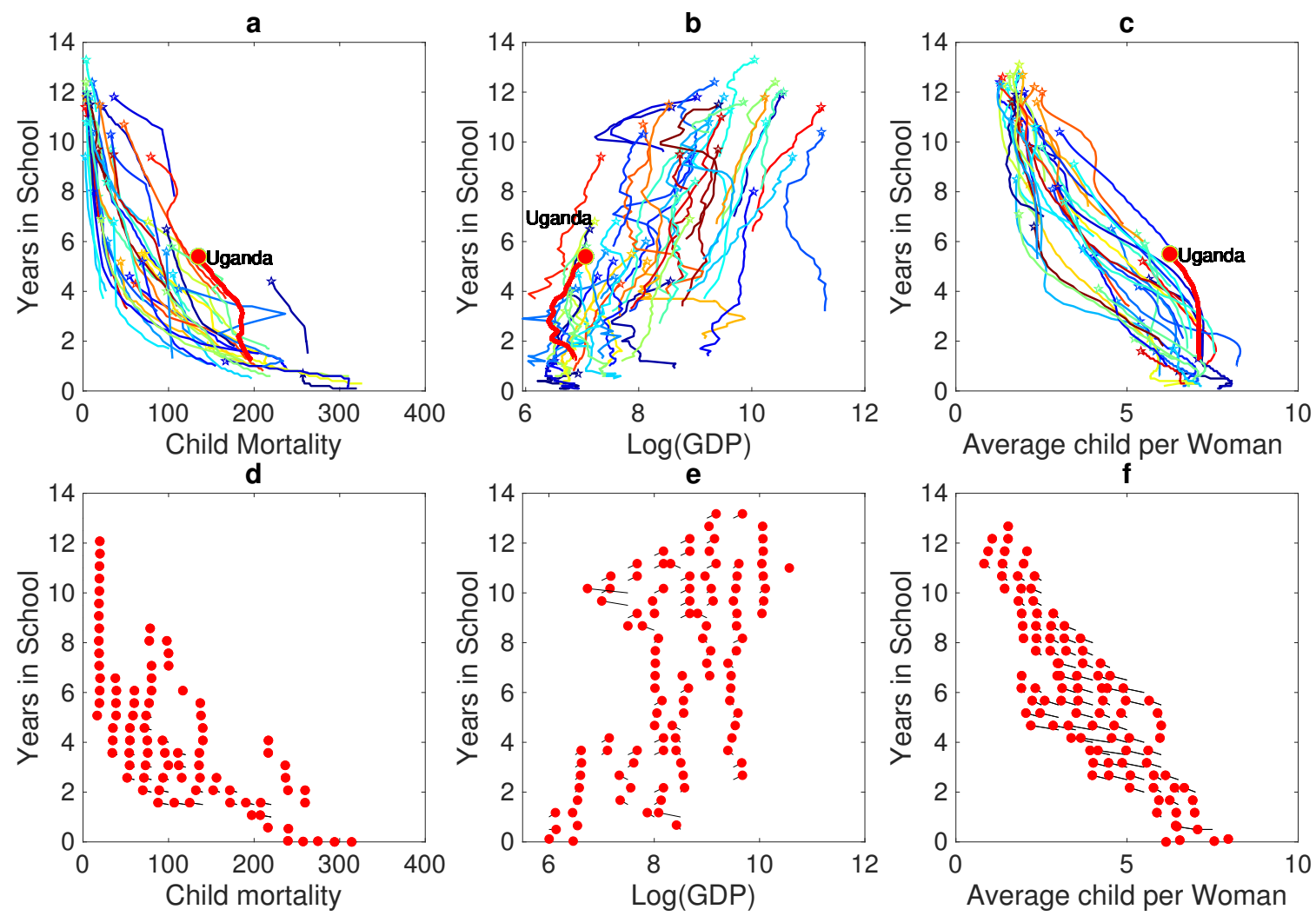

Figure 6: Graph a, b, and c shows data plots of Years in School against child mortality gross domestic product and average child per woman respectively for different countries for a number of years. Uganda is highlighted in bold red. The lines represent the position over time, while the stars show positions over the entire period. Graphs $d$, e and $f$ show vector plots for the indicators generated by all the data

that child mortality reduces, GDP increases, and fertility rates reduce with increasing years in school. Increase in GDP with high education among women indirectly leads to socioeconomic transformation of a nation.

With this revision, and considering that education might not be instant but over a long period

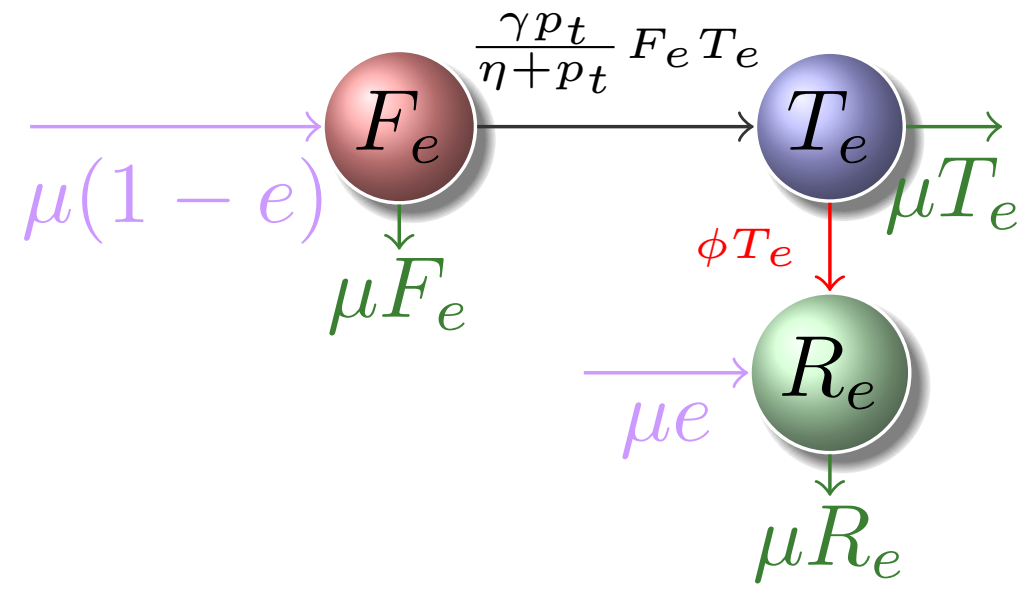

Figure 7: Flux diagram for obstetric fistula in presence of education and demographics

of time to realise benefits, we now include demographics and fistula development rate $\lambda$. 
We let $e \in[0,1]$ be the education level of the women which is in four categories based on the data: Either women have no education (0), have primary education (1/4), secondary education $(2 / 4)$, or post secondary (1). We also include the level of investment $p_{t} \in[0,1]$ which signifies the proportion of the total funds available for maternal care that will be utilised for treatment of fistula women. Parameter $\eta$ is defined as before and $\mu$ is the life expectancy at birth for Ugandan women, which is 56.9 years [46]. To keep the population constant and normalised, we are letting total recruitment rate $\lambda$ be equivalent to the total death rate $\mu$.

The revised model is described in equation (7), and Figure 7, and given as

$$
\begin{aligned}
& \frac{d F_{e}}{d t}=\mu(1-e)-\frac{\gamma p_{t}}{\eta+p_{t}} F_{e} T_{e}-\mu F_{e}, \quad \frac{d T_{e}}{d t}=\frac{\gamma p_{t}}{\eta+p_{t}} F_{e} T_{e}-\phi T_{e}-\mu T_{e}, \\
& \quad \frac{d R_{e}}{d t}=\phi T_{e}-\mu R_{e}+e \mu
\end{aligned}
$$

with solutions $F_{e}^{*}=(1-e) \frac{1}{\mathcal{R}_{e}}, T_{e}^{*}=\frac{\mu\left(\eta+p_{t}\right)}{\gamma p_{t}}\left(\mathcal{R}_{e}-1\right)$, and $R_{e}^{*}=e+\frac{\left(\eta+p_{t}\right)}{p_{t}}\left(\mathcal{R}_{e}-1\right)$ where

$$
\mathcal{R}_{e}=\frac{(1-e) \gamma p_{t} F_{e 0}}{(\mu+\phi)\left(\eta+p_{t}\right)}
$$

and is the threshold hold value for the number of women who should be educated before obstructed labour can be controlled. It is observed from Figure 8 that with increasing proportion
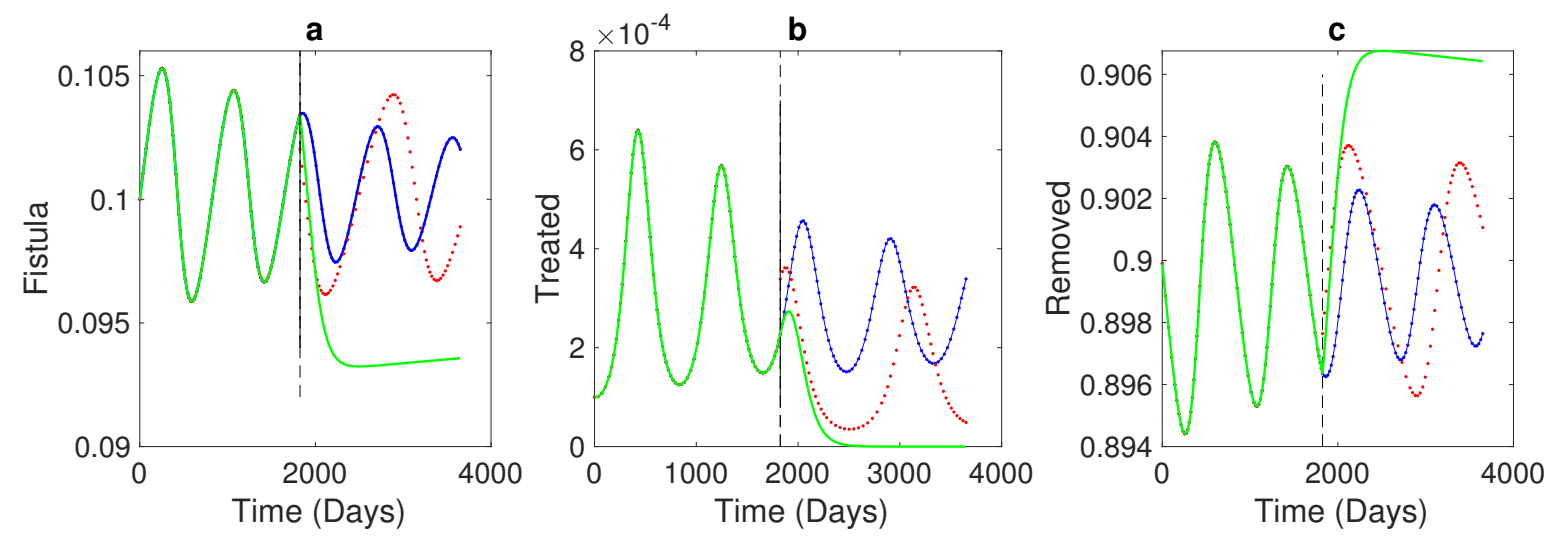

Figure 8: Simulation of the education model with different levels of education $e_{1}=0$ (blue line), $e_{2}=0.5$ (red line) and $e_{3}=1$ (green line). Parameter values used are $\eta=, \mu=1 /(56 * 365)$, and $\phi=1 / 7$. Initial conditions are $F(0)=0.1, T(0)=1 e-04$ and $R(0)=1-F(0)-T(0)$.

of educated women, the number of women that get fistula are fewer and the times between corresponding influxes are longer. When all the women are educated, i.e., $e=1$, we observe that the proportion of women who develop fistula is significantly reduced Figure 8a, and eventually tending to zero as time increases. This observation corresponds to $T(t)$ tending to zero Figure $8 \mathrm{~b}$, and the eventual increase in the removed women Figure 8c. Therefore, offering education to women will help reducing fistula prevalence in Ugandan women.

We can obtain the critical level of education required to reduce fistula cases using equation (8) when $\mathcal{R}_{e}=1$. This gives

$$
e_{\text {critical }}=1-\frac{(\mu+\phi)\left(\eta+p_{t}\right)}{\gamma p_{t} F_{e 0}}
$$


and the relationship between $e_{\text {critical }}$ with increasing values of $p_{t}$ given in Figure 9 . The figure shows that if the proportion of educated women is at least $92.33 \%$, then only $4.5 \%$ could be invested into treatment.

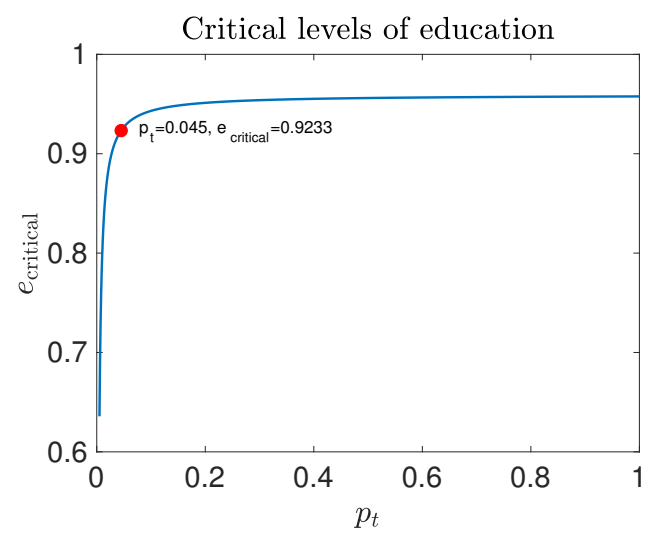

Figure 9: Citical levels of education with investment

\section{Discussion and conclusions}

The data used in the study was from the Uganda Bureau of Statistics, Uganda Demographic Health Survey carried out in fifteen regions of South Central, North Central, Kampala, Busoga, Bukedi, Bugisu, Teso, Karamoja, Lango, Acholi, West Nile, Bunyoro, Tooro, Kigezi and Ankole. It mainly considered the age of the women at firth birth, whether they accessed antenatal care or not during pregnancy, reasons why they did not, whether they had birth at a health facility attended by a skilled provider, the women's level of education, wealth quintile, and their experiences with obstetric fistulas.

The aim of this study was to estimate the burden of obstetric fistula in Uganda and the resulting effect of education of women on its causes. Results showed that high investment rates led to high treatments rates that depended on the level of investment. An optimal investment strategy in treatment was shown to be $44.9 \%$, where for every woman treated, 17 more would opt for treatment. Results also show that educating women reduced fistula prevalence although the magnitude of reduction depends on the level of education coverage. These results show that an optimal strategy to reduce fistula prevalence would require achieving a balance between the two desirable but incompatible health outcomes. This will help maximise the total return or effectiveness under given circumstances such as continued women that develop fistula due to lack of skilled healthcare or lifetime incontinence due to lack of treatment. From this study we conclude that improving access to quality health education will contribute to the elimination of obstetric fistula in Uganda.

This study also examined the association between education level and the prevalence of selfreported obstetric fistula in Ugandan women. To achieve this objective, the women were grouped into three categories: those with fistula symptoms, those that had it and got treated and the recovered. First we considered the case of one cycle without demographics to determine the burden in Uganda. We then repeatedly offer education to women in the reproductive age of $15-49$ to determine how it impacts the overall outcome in comparison to absence 
of education. Since education might take a longer period to realise benefits and its potential in the fight against obstructed labour, demographics are incorporated in the revised model but the population is kept constant. We looked at three scenarios: no education to women, partial education or full education. The education offered is on the causes, dangers, and possible prevention of obstetric fistula. It also provided opportunities for symptomatic women such as treatment for the condition. To optimise health returns, an investment strategy was incorporated which provides the best way to prevent obstructed labour.

For the case of Uganda despite the fact that fistula operations are carried out free of charge in 29 hospitals, from 24 districts [30], even if no new cases occurred, it would take more than 30 years to treat the existing patients at the current rate. All cases of obstetric fistula can be prevented with timely access to emergency obstetric care, especially caesarean section. That it is still a public health problem in Uganda is a result of the egregious failure of the health system to provide safe maternity care. Poor women, without timely access to safe childbirth or delivery care from the onset of labor to the end of the third stage of labor, are most vulnerable to obstetric fistula and the physical and psychological consequences that eventually affect their quality of life.

\section{Acknowledgments}

Betty Nannyonga wishes to acknowledge the financial support from the SIDA bilateral program Makerere 2015-2020 Project Capacity building in mathematics and its application. Further acknowledgement is extended to Linköping University Sweden for hosting the Post Doctorate.

\section{References}

[1] IHME. GBD Heatmap. 2013. Available from: http://vizhub.healthdata.org/ gbd-compare/heatmap. Accessed December 102018.

[2] N.J. Kassebaum et al. Global, regional, and national levels and causes of maternal mortality during 1990-2013: a systematic analysis for the Global Burden of Disease Study 2013. Lancet, 384(9947) 980-1004 (2014).

[3] WHO. Trends in maternal mortality: 1990 to 2013. Estimates by WHO, UNICEF, UNFPA, The World Bank and the United Nations Population Division. Geneva, Switzerland: World Health Organization, 56 (2014).

[4] A. Langer et al. Women and Health: the key for sustainable development. Lancet, 386(9999) 1162-1210 (2015).

[5] S.M. Wilson et al. Psychological Symptoms Among Obstetric Fistula Patients Compared to Gynecology Outpatients in Tanzania. Int J Behav Med, 22(5) 605-613 (2015).

[6] K. Siddle et al. Psychosocial impact of obstetric fistula in women presenting for surgical care in Tanzania. Int Urogynecol J, 24(7) 1215-20 (2013).

[7] L.L. Wall et al. The obstetric vesicovaginal fistula in the developing world. Obstet Gynecol Surv, 60(7 Suppl 1):S3-S51 (2005). 
[8] S. Arrowsmith, E.C. Hamlin, L.L. Wall. Obstructed labor injury complex: obstetric fistula formation and the multifaceted morbidity of maternal birth trauma in the developing world. Obstet Gynecol Surv, 51(9) 568-74 (1996).

[9] J.T. Goh et al. Mental health screening in women with genital tract fistulae. BJOG, 112(9) 1328-30 (2005).

[10] M. Maheu-Giroux, V. Filippi, S. Samadoulougou, M.C. Castro, N. Maulet, N. Meda, F. Kirakoya-Samadoulougou Prevalence of symptoms of vaginal fistula in 19 sub-Saharan Africa countries: a meta-analysis of national household survey data. Lancet Glob Health, 3(5) e271-8 (2015).

[11] A.J. Adler et al. Estimating the prevalence of obstetric fistula: a systematic review and meta-analysis. BMC Pregnancy Childbirth, 13246 (2013).

[12] A.X. Zheng, F.W. Anderson Obstetric fistula in low-income countries. Int J Gynaecol Obstet, 104(2) 85-9 (2009).

[13] M. Muleta, S. Rasmussen, T. Kiserud Obstetric fistula in 14,928 Ethiopian women. Acta Obstet Gynecol Scand, 89(7) 945-51 (2010).

[14] L. Hawkins et al. Characteristics and surgical success of patients presenting for repair of obstetric fistula in western Kenya. Int J Gynaecol Obstet, 120(2) 178-82 (2013).

[15] P.M. Tebeu PM et al. Risk factors for obstetric fistula: a clinical review. Int Urogynecol J, 23(4) 387-94 (2012).

[16] M.A. ljaiya et al. Vesicovaginal fistula: a review of Nigerian experience. West Afr J Med, 29(5) 293-8 (2010).

[17] A. Holme, M. Breen, C. MacArthur Obstetric fistulae: a study of women managed at the Monze Mission Hospital, Zambia. BJOG, 114(8) 1010-7 (2007).

[18] E. Landry et al. Profiles and experiences of women undergoing genital fistula repair: findings from five countries. Glob Public Health, 8(8) 926-42 (2013).

[19] L.L Wall et al. The obstetric vesicovaginal fistula: characteristics of 899 patients from Jos, Nigeria. Am J Obstet Gynecol, 190(4) 1011-9 (2004).

[20] F. Tahzib Epidemiological determinants of vesicovaginal fistulas. Br J Obstet Gynaecol, 90(5) 387-91 (1983).

[21] L.T. Mselle et al. Waiting for attention and care: birthing accounts of women in rural Tanzania who developed obstetric fistula as an outcome of labour. BMC Pregnancy Childbirth, 1175 (2011).

[22] G.S: Melah et al. Risk factors for obstetric fistulae in north-eastern Nigeria. J Obstet Gynaecol,, 27(8) 819-23 (2007).

[23] Z. G. Roka et al. Factors associated with obstetric fistulae occurrence among patients attending selected hospitals in Kenya, 2010: a case control study. BMC Pregnancy Childbirth,13 56 (2013). 
[24] J. K Barageine et al. Risk factors for obstetric fistula in Western Uganda: a case control study. PLoS One, 9(11) e112299 (2014).

[25] A. Browning, A. Lewis, S. Whiteside Predicting women at risk for developing obstetric fistula: a fistula index? An observational study comparison of two cohorts. BJOG, 121(5) 604-9 (2014).

[26] A.M. Norman, M. Breen, H. E. Richter Prevention of obstetric urogenital fistulae: some thoughts on a daunting task. Int Urogynecol J Pelvic Floor Dysfunct, 18(5) 485-91 (2007).

[27] L. L. Wall Overcoming phase 1 delays: the critical component of obstetric fistula prevention programs in resource-poor countries. BMC Pregnancy Childbirth, 1268 (2012).

[28] J. M. Turan, K. Johnson, M. L. Polan Experiences of women seeking medical care for obstetric fistula in Eritrea: implications for prevention, treatment, and social reintegration. Glob Public Health, 2(1) 64-77 (2007).

[29] S. Miller, F. Lester, M. Webster, B. Cowan Obstetric fistula: a preventable tragedy. J Midwifery \& Women's Health. 50 286-294 (2005).

[30] Obstetric fistula leaves a million women needlessly incontinent, too often outcast - modern-day lepers. https://www.fistulafoundation.org/. Accessed August 10 2017.

[31] A. A. Creanga, R. R. Genadry Obstetric fistulas: A clinical review. International Journal of Gynecology \& Obstetrics, 99 Suppl 1 S40-6 (2007).

[32] Rectovaginal Fistula. Mayo Clinic. Mayo Foundation for Medical Education and Research, 29 May 2010. http://www.mayoclinic.com/health/rectovaginal-fistula/ DS01065/DSECTION=symptoms. Accessed August 112017.

[33] S. Ahmed, S. A. Holtz Social and economic consequences of obstetric fistula: Life changes forever? International Journal of Gynecology \& Obstetrics, 99 S10-S15 (2007).

[34] B. J. Champagne, M. F. McGee Rectovaginal fistula. The Surgical Clinics of North America, 90 69-82 (2010).

[35] J. M. Novi, D. O. Northington, M. Gina Rectovaginal fistula. Journal of Pelvic Medicine \& Surgery, 11 283-293 (2005).

[36] M. Wong, B. Ozel Fistulae. In Management of Common Problems in Obstetrics and Gynecology, 5 328-332. Chichester: Wiley-Blackwell (2010).

[37] M. Bangser Strengthening public health priority-setting through research on fistula, maternal health, and health inequities. Int J Gynaecol Obstet, 99(Suppl 1) 16-20 (2007).

[38] N. Kasamba, D. K. Kaye, S. N. Mbalinda Community awareness about risk factors, presentation and prevention and obstetric fistula in Nabitovu village, Iganga district Uganda. BMC Pregnancy Childbirth, 13229 (2013).

[39] L. T. Mselle, K. M. Moland, B. Evjen-Olsen, A. Mvungi, T. W. Kohi "I am nothing": experiences of loss among women suffering from severe birth injuries in Tanzania. BMC Womens Health, 1149 (2011). 
[40] M. Kayondo, S. Wasswa, J. Kabakyenga, N. Mukiibi, J. Senkungu, A. Stenson, P. Mukasa. Predictors and outcome of surgical repair of obstetric fistula at a regional referral hospital, Mbarara, western Uganda. BMC Urol, 1123 (2011).

[41] S. Ahmed, S. A. Holtz Social and economic consequences of obstetric fistula: life changed forever? Int J Gynaecol Obstet, 99(Suppl 1) 10-5 (2007).

[42] S. Ramphal, J. Moodley Vesicovaginal fistula: obstetric causes. Curr Opin Obstet Gynecol, 18(2) 147-51 (2006).

[43] L. Semere, N. M. Nour Obstetric fistula: Living with incontinence and shame. Reviews in Obstetrics \& Gynecology, 1(4) 193-197 (2008).

[44] R. Mathur, N. Joshi, G. Aggarwal, R. Raikwar, V. Shrivastava, P. Mathur, P. Raikwar, R. Joshi Urogenital fistulae: A prospective study of 50 cases at a tertiary care hospital. Urol Ann, 2(2) 67-70 (2010).

[45] M. Walubiri, H. Sekanjako https://www.newvision.co.ug/new_vision/news/ 1486736/75-ugandan-women-suffer-obstetric-fistula. Accessed December 10, 2018.

[46] Uganda Population, (1950-2017). http://www.worldometers.info/ world-population/uganda-population/. Accessed August 102017.

[47] M. Maheu-Giroux, V. Filippi, S. Samadoulougou, M. C. Castro, N. Maulet, N. Meda, F. Kirakoya-Samadoulougou Prevalence of symptoms of vaginal fistula in 19 sub-Saharan Africa countries: a meta-analysis of national household survey data. Lancet Glob Health, 3(5) e271-8 (2015). 